\title{
PRODUÇÃO, TEOR DE BORO E AVALIAČ̃̃O DE EXTRATỎRES EM SOLOS CULTIVADOS COM SOJA
}

\author{
Yield, boron concentration and evaluation of extractants in soils cultivated with soybean
}

\author{
Adônis Moreira ${ }^{1}$, César de Castro², Fábio Alvares de Oliveira $^{2}$
}

\begin{abstract}
RESUMO
Neste trabalho, objetivou-se avaliar a eficiência de seis extratores de B e o efeito de doses sobre os teores de B e produção de matéria seca da soja cultivada em condições de casa de vegetação com LATOSSOLO VERMELHO Eutroférrico coletados em Mauá da Serra e Londrina (PR) e NEOSSOLO QUARTZARÊNICO Órtico de Alto Garças (MT). O delineamento experimental utilizado foi o inteiramente casualizado, constituído por seis doses de B $\left(0,0,25,0,5,1,2 \mathrm{e} 4 \mathrm{~kg} \mathrm{ha}^{-1}\right)$, com três repetições. O B disponível foi determinado por seis soluções extratoras: Mehlich 1, Mehlich 3, água quente, $\mathrm{HCl} \mathrm{0,05} \mathrm{mol} \mathrm{L-1}, \mathrm{Ca}\left(\mathrm{H}_{2} \mathrm{PO}_{4}\right)_{2} \cdot \mathrm{H}_{2} \mathrm{O} 0,01 \mathrm{~mol} \mathrm{~L}^{-1} \mathrm{e} \mathrm{KCl} 1,0 \mathrm{~mol} \mathrm{~L}^{-1}$. Nas condições estudadas, os resultados mostraram que o $\mathrm{B}$ extraído por água quente acarretou as melhores correlações com a produção de matéria seca e conteúdo de B na planta, enquanto o extrator $\mathrm{KCl} 1,0 \mathrm{~mol} \mathrm{~L}^{-1}$ foi o mais eficiente na determinação do teor de $\mathrm{B}$ no tecido vegetal. Altas concentrações de B diminuíram a eficiência do extrator água quente em determinar o B disponível do solo. As maiores correlações foram obtidas entre os extratores Mehlich 1 e Ca( $\left.\mathrm{H}_{2} \mathrm{PO}_{4}\right)_{2} \cdot \mathrm{H}_{2} \mathrm{O} 0,01 \mathrm{~mol} \mathrm{~L}^{-1}, \mathrm{KCl} 1,0 \mathrm{~mol} \mathrm{~L}^{-1}$ e $\mathrm{HCl} 0,05 \mathrm{~mol} \mathrm{~L}^{-1}, \mathrm{KCl} 1,0 \mathrm{~mol} \mathrm{~L}^{-1}$ e Ca$\left(\mathrm{H}_{2} \mathrm{PO}_{4}\right)_{2} \cdot \mathrm{H}_{2} \mathrm{O}$ $0,01 \mathrm{~mol} \mathrm{~L}^{-1}$ e água quente e $\mathrm{Ca}\left(\mathrm{H}_{2} \mathrm{PO}_{4}\right)_{2} \cdot \mathrm{H}_{2} \mathrm{O} 0,01 \mathrm{~mol} \mathrm{~L}^{-1}$.
\end{abstract}

Termos para indexação: Glycine max, métodos de análise de solo, micronutriente, disponibilidade de nutriente.

\begin{abstract}
The objective of this work was to evaluate the efficiency of six extractants and the effect of boron concentration and dry matter yield, cultivated in greenhouse conditions with two Rhodic Hapludox in Mauá da Serra and Londrina, in the State of Paraná, and one Ustoxix Quatzipsamment in Alto Garças, State of Mato Grosso, Brazil. The experiment was conducted in a completely randomized design with six boron rates $\left(0,0.25,0.5,1,2\right.$ and $\left.4 \mathrm{~kg} \mathrm{ha}^{-1}\right)$, and three replicates. The available boron was determined with six extractant solutions: Mehlich 1, Mehlich 3, hot water, $\mathrm{HCl} 0.05 \mathrm{~mol} \mathrm{~L}^{-1}, \mathrm{Ca}\left(\mathrm{H}_{2} \mathrm{PO}_{4}\right)_{2} \cdot \mathrm{H}_{2} \mathrm{O} 0.01 \mathrm{~mol} \mathrm{~L}^{-1}$ and $\mathrm{KCl} 1.0 \mathrm{~mol} \mathrm{~L} \mathrm{~L}^{-1}$. The results showed that the $\mathrm{B}$ extracted by hot water had the best correlation with the dry matter yield and B content in the plant, whereas the $\mathrm{KCl} 1.0 \mathrm{~mol} \mathrm{~L}^{-1}$ was the most efficient in the determination of $\mathrm{B}$ level in dry matter. High concentrations of $\mathrm{B}$ diminished the efficiency of the hot water extractant in determining the $\mathrm{B}$ available in soil. The highest correlations were obtained between the extractant Mehlich 1 and $\mathrm{Ca}_{(}\left(\mathrm{H}_{2} \mathrm{PO}_{4}\right)_{2} \cdot \mathrm{H}_{2} \mathrm{O} 0.01 \mathrm{~mol} \mathrm{~L}^{-1}, \mathrm{KCl} 1.0$ $\mathrm{mol} \mathrm{L}-1$ and $\mathrm{HCl} 0.05 \mathrm{~mol} \mathrm{~L}^{-1}, \mathrm{KCl} 1.0 \mathrm{~mol} \mathrm{~L}^{-1}$ and $\mathrm{Ca}\left(\mathrm{H}_{2} \mathrm{PO}_{4}\right)_{2} \cdot \mathrm{H}_{2} \mathrm{O} 0.01 \mathrm{~mol} \mathrm{~L}^{-1}$, and hot water and $\left.\mathrm{Ca}_{2}^{2}\left(\mathrm{H}_{2} \mathrm{PO}_{4}\right)_{2}\right)_{2} \cdot \mathrm{H}_{2} \mathrm{O} 0.01 \mathrm{~mol} \mathrm{~L}^{-1}$.
\end{abstract}

Index terms: Glycine max, methods of soil analysis, micronutrient, nutrient availability.

(Recebido em 10 de agosto de 2007 e aprovado em 1 de julho de 2009)

\section{INTRODUÇÃO}

Os testes de calibração e avaliação são necessários para um programa de recomendação de adubação, sem essas informações, os teores obtidos no solo e nas plantas têm pouco significado. Para isso, soluções extratoras têm sido desenvolvidas e apresentando resultados satisfatórios em identificar o grau de suficiência de dado elemento, definindo a quantidade que o mesmo pode ser aplicado (Evans, 1987).

No caso do B, atualmente nos laboratórios de fertilidade do solo, a determinação da sua disponibilidade é feita em água quente sob refluxo ou assistido por microondas (Gupta, 1967; Abreu et al., 2001). Apesar das modificações, o método adaptado para utilização do microondas necessita de aparelho com potência específica, enquanto no refluxo, existe a dificuldade no controle preciso da temperatura nos procedimentos de aquecimento e resfriamento da suspensão solo-extrator, aliado à dificuldade de limpeza e à exigência de vidrarias isentas de borossilicatos.

Além dessas dificuldades, o principal problema do determinado método tem sido, em alguns casos, a baixa precisão do diagnóstico, uma vez que não está sendo capaz de separar adequadamente dentro do grupo de solos com teores diversos de $\mathrm{B}$, aqueles que realmente apresentam baixa disponibilidade e que resultarão em baixos ou tóxicos teores foliares, ocasionando baixa produtividade, como atualmente visto em áreas de Cerrado, em especial, nos estados de Mato Grosso e Goiás, especialmente, com plantas indicadoras de B, como no caso do girassol (Castro \& Oliveira, 2005).

Outros métodos de extração estão sendo frequentemente propostos e comparados com água quente sob refluxo ou aquecido com microondas, tais como: Mehlich 1, Mehlich 3, $\mathrm{HCl} 0,05 \mathrm{~mol} \mathrm{~L}^{-1}, \mathrm{HCl} 0,1 \mathrm{~mol} \mathrm{~L}^{-1}, \mathrm{CaCl}_{2} 0,01 \mathrm{~mol} \mathrm{~L}^{-1}$,

${ }^{1}$ Embrapa Pecuária Sudeste/CPPSE - Cx. P. 339 - 13560-970 - São Carlos, SP - adonis@cppse.embrapa.br

${ }^{2}$ Embrapa Soja/CNPSO - Londrina, PR 
$\mathrm{CaCl}_{2}$ 0,05 mol L-1, entre outros. O estudo dessas soluções é justificado pelo baixo custo da extração e pela simplicidade em comparação com água quente ou suas adaptações (Bataglia \& Raij, 1990). Mesmo com essas vantagens, no caso dos extratores ácidos que extraem quantidades significativas de Fe, interferindo na determinação do B disponível do solo.

Conduziu-se este trabalho, com o objetivo de estudar a eficiência de seis extratores [ $\mathrm{H}_{2} \mathrm{O}$ quente, $\mathrm{KCl}$ 1,0 $\mathrm{mol} \mathrm{L}^{-1}$,

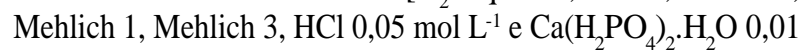
mol L ${ }^{-1}$ ] na determinação do $\mathrm{B}$ disponível no solo, bem como o efeito de doses na disponibilidade do B em solos cultivados com soja com características químicas e físicas distintas.

\section{MATERIAL E MÉTODOS}

O experimento foi realizado no período de 2005, em vasos de plásticos com 3,0 kg de capacidade sob condições de casa de vegetação da Embrapa Soja, Londrina, PR. Os solos foram coletados na camada de $0-20 \mathrm{~cm}$ nos municípios de Alto Garças (MT) - NEOSSOLO QUARTZARÊNICO Órtico, NQo, Mauá da Serra (PR) - LATOSSOLO VERMELHO Eutroférrico, LVef e Londrina (PR) LATOSSOLO VERMELHO Eutroférrico, LVef, cujas características são apresentadas na Tabela 1.

Empregou-se o delineamento inteiramente casualizado, com três repetições. Os tratamentos foram constituídos das doses $0,0,25,0,5,1,0,2,4 \mathrm{~kg} \mathrm{ha}^{-1}$ de B equivalendo a $0,0,125,0,25,0,5,1$ e $2 \mathrm{mg} \mathrm{kg}^{-1}$ (fonte - ácido bórico) aplicados na forma de solução e misturados no solo. $\mathrm{Na}$ determinação do $\mathrm{B}$ disponível, foram utilizados os extratores $\mathrm{H}_{2} \mathrm{O}$ quente (B-AQ) (Abreu et al., 2001), $\mathrm{KCl}$ 1,0 mol L-1 (B-KCl) (Moreira \& Castro, 2006), Mehlich 1 (B-M1) (Mehlich, 1978), Mehlich 3 (B-M3) (Mehlich, 1984), HCl 0,05 mol L-1 (B-HCl) (Ponnamperuma et al., 1981) e $\mathrm{Ca}\left(\mathrm{H}_{2} \mathrm{PO}_{4}\right)_{2} \cdot \mathrm{H}_{2} \mathrm{O}$ $0,01 \mathrm{~mol} \mathrm{~L}^{-1}\left(\mathrm{~B}^{-\mathrm{CaPO}_{4}}\right)$ (Alvarez Venegas et al., 2001). Exceto o extrator $\mathrm{H}_{2} \mathrm{O}$ quente, a relação solo e solução extratora dos demais métodos foi de 1:10 (v:v). O teor de B na parte aérea foi feito por incineração a $500{ }^{\circ} \mathrm{C}$ e determinado com reagente colorimétrico Azometina-H, no comprimento de onda de 420 nm (Malavolta et al., 1997; Abreu et al., 2001).

Para a caracterização da biodisponibilidade do B, foram cultivadas, após o desbaste, quatro plantas de soja 'BRS 132'. Os vasos foram mantidos com umidade ao redor de $70 \%$ do VTP (valor total de poros), por meio de reposição diária com água destilada.

Trinta dias antes do plantio, foi aplicado calcário dolomítico para elevar a saturação por bases a $70 \%$ (Tecnologias de Produção de Soja, 2006). Exceto o N, inoculado com o Bradyrhizobium japonicum e o B que fez parte dos tratamentos, a adubação básica em todos os vasos consistiu na aplicação de $100 \mathrm{mg} \mathrm{kg}^{-1}$ de $\mathrm{P}_{2} \mathrm{O}_{5}$ (superfosfato triplo - 41\% de $\left.\mathrm{P}_{2} \mathrm{O}_{5}\right), 150 \mathrm{mg} \mathrm{kg}^{-1}$ de $\mathrm{K}(\mathrm{KCl}-58 \%$ de $\mathrm{K}), 2,0$ $\mathrm{mg} \mathrm{kg}^{-1}$ de $\mathrm{Zn}\left(\mathrm{ZnSO}_{4} .7 \mathrm{H}_{2} \mathrm{O}-20 \%\right.$ de $\left.\mathrm{Zn}\right)$ e $25,0 \mathrm{mg} \mathrm{kg}^{-1}$ de $\mathrm{Mn}\left(\mathrm{MnCl}_{2} \cdot 4 \mathrm{H}_{2} \mathrm{O}-35 \%\right.$ de $\left.\mathrm{Mn}\right)$ (Malavolta, 1980).

A colheita da parte aérea foi realizada no início do florescimento no estádio R1 (Fehr \& Caviness, 1971). Depois de coletado, o material foi seco em estufa com circulação forçada até peso constante. Posteriormente, pesado para determinação da matéria seca da parte aérea (MSPA) e moído para determinação do teor de B total na parte aérea da soja.

Os resultados foram submetidos à análise de variância (ANOVA), teste $\mathrm{F}$, regressão e correlação a 5\% de significância, conforme metodologias descritas por Pimentel Gomes \& Garcia (2002).

\section{RESULTADOS E DISCUSSÃO}

De acordo com as equações de regressão (Figura 1), os teores estimados de B disponível, após a produção de matéria seca da parte aérea nos três solos, variaram na média de 0,65 a 1,27 $\mathrm{mg} \mathrm{kg}^{-1}$ com o extrator água quente, de 0,16 a $0,21 \mathrm{mg} \mathrm{kg}^{-1}$ com o $\mathrm{HCl} 0,05 \mathrm{~mol} \mathrm{~L}^{-1}$, de 0,19 a $0,28 \mathrm{mg} \mathrm{kg}^{-1}$ com $\mathrm{Ca}\left(\mathrm{H}_{2} \mathrm{PO}_{4}\right)_{2} \cdot \mathrm{H}_{2} \mathrm{O} 0,01 \mathrm{~mol} \mathrm{~L}^{-1}$, e de 0,06 a $0,31 \mathrm{mg} \mathrm{kg}^{-1}$ com o $\mathrm{KCl} 1,0 \mathrm{~mol} \mathrm{~L}^{-1}$, enquanto os extratores Mehlich 1 e Mehlich 3 não apresentaram significância $(\mathrm{p}<0,05)$. Observou-se, também, que os altos teores de B disponível nos solos antes do plantio e aplicação dos tratamentos, durante a condução do experimento ocasionaram em sintomas visuais de toxidez de B nas plantas no NEOSSOLO QUARTZARÊNICO Órtico de Alto Garças (Rqo-AG) e no LATOSSOLO VERMELHO Eutroférrico de Mauá da Serra (LVdf-MS).

As produções de MSPA e os seus respectivos teores de B são mostrados na Tabela 2. Verificou-se que, apesar dos teores iniciais nos três solos estarem dentro das faixas de interpretação considerada média e alta (Tecnologias de Produção de Soja, 2006), no RQo (90,0 g $\mathrm{kg}^{-1}$ de argila e 15,0 $\mathrm{g} \mathrm{kg}^{-1}$ de MO) e LVef-MS (736,0 $\mathrm{g} \mathrm{kg}^{-1}$ de argila e 52,6 $\mathrm{g} \mathrm{kg}^{-1}$ de MO), a aplicação de $0,5 \mathrm{~kg} \mathrm{ha}^{-1}$ de $\mathrm{B}\left(0,125 \mathrm{mg} \mathrm{kg}^{-1}\right.$ e $\left.31,3 \mathrm{~g} \mathrm{~kg}^{-1} \mathrm{de} \mathrm{MO}\right)$, proporcionou as maiores produções de matéria seca, enquanto no LVef-Lo de Londrina, (776,0 $\mathrm{g} \mathrm{kg}^{-1}$ e 31,3 $\mathrm{g} \mathrm{kg}^{-1}$ de MO) foi com 2,0 $\mathrm{kg} \mathrm{ha}^{-1}$, porém, esse valor não apresentou diferenças significativas com as demais doses de B (Tabela 2).

Os extratores ácidos tiveram em cada solo, ordenamentos distintos das doses sobre os teores de B na matéria seca, apresentando, em sua maioria, a seguinte ordem de disponibilidade: LVef-MS $>$ Rqo-AG $>$ LVef-Lo (Figura 1). Observou-se, no entanto, que o extrator Mehlich 3 foi o que separou mais uniformemente os teores em cada solo estudado (Rqo-AG - $\hat{y}=$ $0,691+0,208^{*} \mathrm{x}, \mathrm{r}=0,98$; LVef-MS $-\hat{y}=0,425+0,073^{*} \mathrm{x}, \mathrm{r}$ $=0,95$ e LVef-Lo $-\hat{y}=0,053+0,054 * x, r=0,95)$. 
Tabela 1 - Composição química e física dos solos ${ }^{1}$.

\begin{tabular}{|c|c|c|c|}
\hline \multirow[t]{2}{*}{ Propriedades } & \multicolumn{3}{|c|}{ Solos } \\
\hline & LVef-Lo & LVef-MS & RQo-AG \\
\hline $\mathrm{pH}\left(\mathrm{CaCl}_{2} 0,01 \mathrm{~mol} \mathrm{~L}^{-1}\right)$ & 4,9 & 5,3 & 6,2 \\
\hline Matéria orgânica - MO $\left(\mathrm{g} \mathrm{kg}^{-1}\right)$ & 31,3 & 56,2 & 15,0 \\
\hline $\mathrm{P}\left(\mathrm{mg} \mathrm{kg}^{-1}\right)$ & 37,3 & 6,7 & 2,7 \\
\hline $\mathrm{K}\left(\mathrm{cmol}_{\mathrm{c}} \mathrm{dm}^{-3}\right)$ & 0,7 & 0,2 & 0,1 \\
\hline $\mathrm{Ca}\left(\mathrm{cmol}_{\mathrm{c}} \mathrm{dm}^{-3}\right)$ & 5,1 & 3,8 & 1,1 \\
\hline $\mathrm{Mg}\left(\mathrm{cmol}_{\mathrm{c}} \mathrm{dm}^{-3}\right)$ & 1,9 & 3,6 & 1,0 \\
\hline $\mathrm{S}\left(\mathrm{mg} \mathrm{kg}^{-1}\right)$ & 79,1 & 30,1 & 2,7 \\
\hline $\mathrm{Al}\left(\mathrm{cmol}_{\mathrm{c}} \mathrm{dm}^{-3}\right)$ & 0,1 & 0,0 & 0,0 \\
\hline $\mathrm{H}+\mathrm{Al}\left(\mathrm{cmol}_{\mathrm{c}} \mathrm{dm}^{-3}\right)$ & 3,8 & 4,4 & 2,0 \\
\hline CTC $\left(\mathrm{cmol}_{\mathrm{c}} \mathrm{dm}^{-3}\right)$ & 11,5 & 12,0 & 5,2 \\
\hline Saturação por bases (V\%) & 67,0 & 63,3 & 52,4 \\
\hline $\mathrm{S}\left(\mathrm{mg} \mathrm{kg}^{-1}\right)$ & 79,1 & 30,1 & 2,7 \\
\hline $\mathrm{B}\left(\mathrm{mg} \mathrm{kg}^{-1}\right)$ & 0,6 & 0,5 & 0,4 \\
\hline $\mathrm{Cu}\left(\mathrm{mg} \mathrm{kg}^{-1}\right)$ & 18,3 & 4,9 & 0,5 \\
\hline $\mathrm{Fe}\left(\mathrm{mg} \mathrm{kg}^{-1}\right)$ & 101,6 & 156,4 & 150,0 \\
\hline $\operatorname{Mn}\left(\mathrm{mg} \mathrm{kg}^{-1}\right)$ & 173,8 & 28,7 & 33,0 \\
\hline $\mathrm{Zn}\left(\mathrm{mg} \mathrm{kg}^{-1}\right)$ & 7,9 & 6,2 & 1,2 \\
\hline Areia $\left(\mathrm{g} \mathrm{kg}^{-1}\right)$ & 120,0 & 281,0 & 894,0 \\
\hline $\operatorname{Argila}\left(\mathrm{g} \mathrm{kg}^{-1}\right)$ & 710,0 & 614,0 & 90,0 \\
\hline
\end{tabular}

${ }^{1}$ Metodologia de Análise - Embrapa (1997). LVef-Lo - LATOSSOLO VERMELHO Eutroférrico de Londrina (PR), LVef-MS LATOSSOLO VERMELHO Eutroférrico de Mauá da Serra e RQo-AG - NEOSSOLO QUARTZARÊNICO Órticos de Alto Garças (MT) (PR).

No caso da água quente, a melhor relação para o efeito das doses sobre a disponibilidade de B foi com a equação do segundo grau. Tal inferência concorda com as preocupações de Castro \& Oliveira (2005), sobre a baixa capacidade desse extrator de separar adequadamente dentro do grupo de solos com altos teores de B a real disponibilidade do nutriente. Tal fato pode se tornar preocupante, haja vista, ser o micronutriente mais comercializado no Brasil, independentemente de existir ou não a sua real necessidade avaliada pela análise de solo ou na análise foliar (Yamada, 2000), e da sua aplicação ser feita rotineiramente, sem levar em consideração as reais exigências das culturas.

Esse resultado com a água quente, também pode ocasionar em dificuldades na definição do nível crítico, ou na determinação da faixa de suficiência de B para a cultura da soja, que são usadas na tomada de decisão de recomendação de adubação. Cabe esclarecer que a prática da adubação poder representar $50 \%$ do custo variável de produção, podendo aumentar significativamente essa porcentagem, se a mesma for feita inadequadamente.
Nos LVef-MS e Rqo-AG, a aplicação de quantidades superiores a $0,5 \mathrm{~kg} \mathrm{ha}^{-1}$ de $\mathrm{B}$ reduziu significativamente a produção de matéria seca, fato esse, não observado no LVef-Lo (Tabela 2). Com relação ao teor de B na soja, houve efeito linear positivo das doses, inclusive no tratamento sem B, o que é justificado pela menor capacidade de adsorção do NEOSSOLO QUARTZARÊNICO Órtico em razão da textura arenosa e baixo teor de MO (Tabela 2).

A soja cultivada no LVef de Londrina não apresentou correlação significativa entre a produção e o teor de B $\left(0,47^{\text {ns }}-Q\right)$, enquanto no LVef de Mauá da Serra e no RQo de Alto Garças, as correlações foram significativas com coeficientes de correlação de $0,61^{*}$ (L) e $0,81 *(L)$, respectivamente. Segundo Goldberg (1997), a matéria orgânica é o principal constituinte do solo que afeta a disponibilidade de B para as plantas, $\mathrm{o}$ mesmo ocorre com a presença de outros ânions e do teor de argila do solo. $\mathrm{O}$ mesmo autor ressalta que a presença do material orgânico também pode reativar o B ocluso adsorvido nas argilas e nos solos, aumentando o B disponível do solo. 
$\mathrm{O}$ teor de $\mathrm{B}$ na matéria seca variou de 59,22 a $236,45 \mathrm{mg} \mathrm{kg}^{-1}$ no Rqo-AG, de 42,23 a 213,52 $\mathrm{mg} \mathrm{kg}^{-1}$ no LVef-MS, e de 26,10 a 76,03 mg kg-1 no LVef-Lo (Tabela 2), e tiveram correlações significativas $(p \leq 0,05)$ com todos
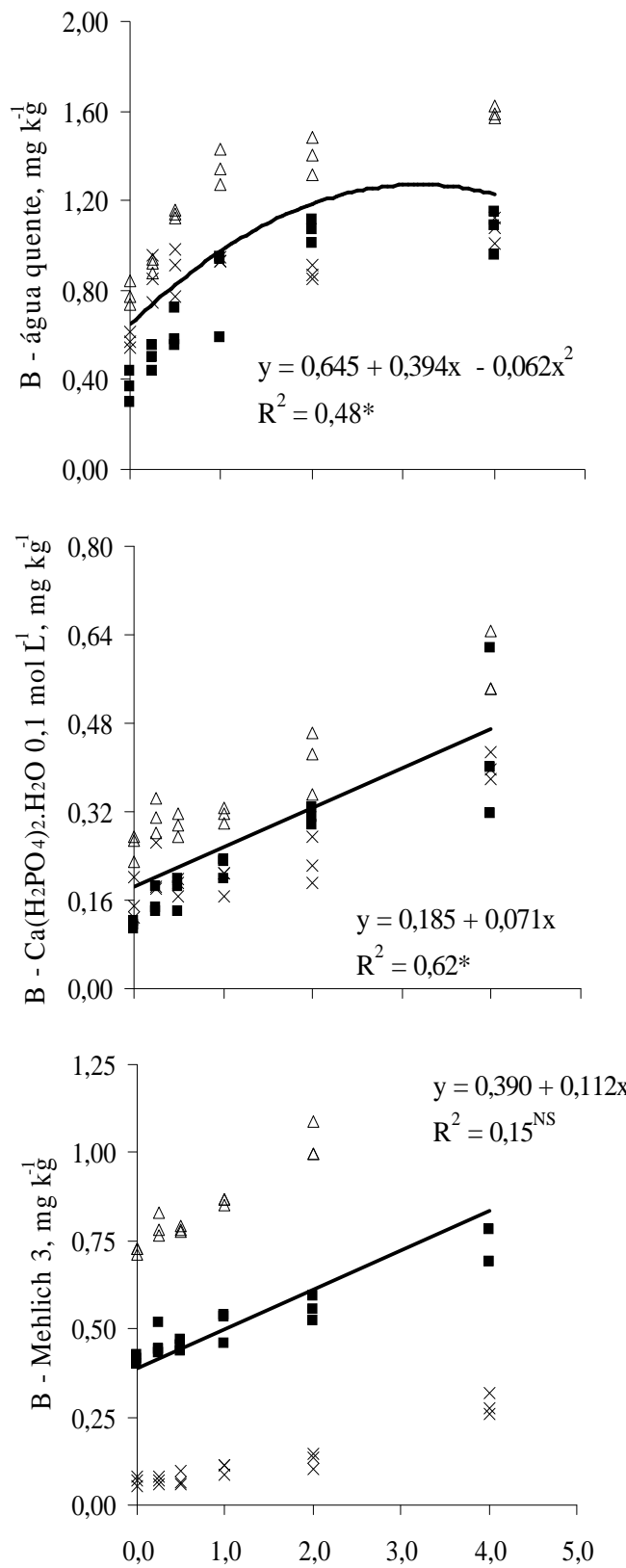

os extratores estudados (Tabela 3). Mesmo na testemunha, os teores ficaram dentro ou bem acima da faixa de 21 a $55 \mathrm{mg} \mathrm{kg}^{-1}$ considerada adequada por Malavolta et al. (1997).
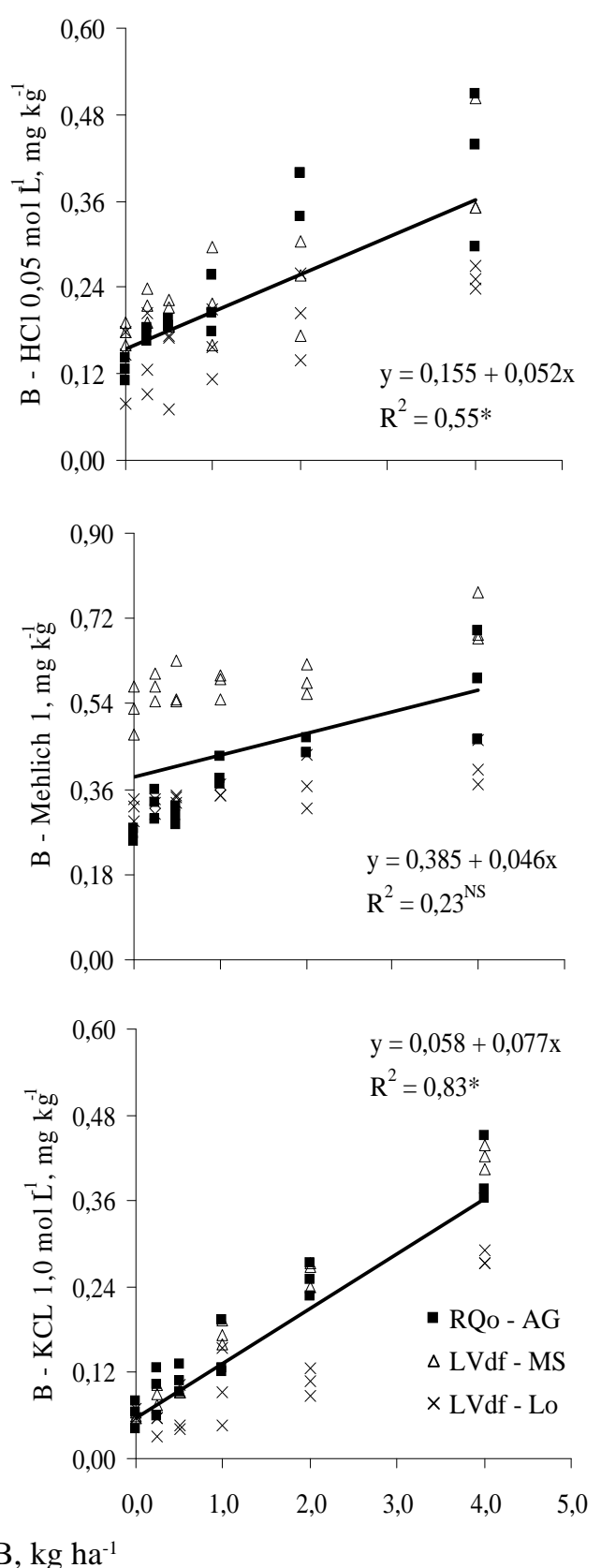

Figura 1 - Relação entre as doses com a disponibilidade de $\mathrm{B}$ obtida com os extratores $\mathrm{H}_{2} \mathrm{O}$ quente, $\mathrm{HCl} 0,05 \mathrm{~mol} \mathrm{~L}^{-1}$, $\mathrm{Ca}\left(\mathrm{H}_{2} \mathrm{PO}_{4}\right)_{2} \cdot \mathrm{H}_{2} \mathrm{O}$ 0,01 mol L-1 , Mehlich 1, Mehlich 3, e KCl 1,0 mol L-1. Rqo de Alto Garças (MT); LVef-MS e LVef -Lo de Mauá da Serra e de Londrina $(\mathrm{PR})$, respectivamente. * significativo a $5 \%$ e NS não significativo. 
Comparando-se o B disponível para cada extrator com a produção de matéria seca, teor e conteúdo de B na soja, com exceção do LATOSSOLO VERMELHO Eutroférrico (Londrina), verificou-se que, em razão da tendência quadrática da curva em função das doses, o B extraído por água quente acarretou nas melhores correlações com a produção de matéria seca e conteúdo de $\mathrm{B}$ na planta, enquanto o extrator $\mathrm{KCl} 1,0 \mathrm{~mol} \mathrm{~L}^{-1}$ foi o mais eficiente em função das doses (Figura 1) e na determinação do teor de B na soja (Tabela 3).

Tais resultados corroboram Silva \& Ferreyra (1998) com o extrator água quente, em estudo realizado com 29 amostras de solo do Estado do Ceará e os obtidos por
Moreira \& Castro (2006), com o extrator $\mathrm{KCl}$ 1,0 mol L-1 em Latossolo Amarelo distrófico do Estado do Amazonas.

No conjunto de todos os solos e dos tratamentos, exceto o extrator Mehlich 3, que correlacionou significativamente somente com $\mathrm{HCl} 0,05 \mathrm{~mol} \mathrm{~L}^{-1}$, nos demais houve correlação significativa $(\mathrm{p} \leq 0,05)$ entre os extratores. As maiores correlações foram entre os extratores B-Mehlich

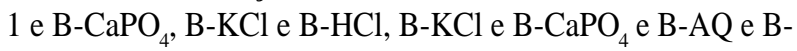
$\mathrm{CaPO}_{4}$ (Tabela 4). Bataglia \& Raij (1990), Silva \& Ferreyra (1998) e Moreira \& Castro (2006), também encontraram correlações significativas e positivas entre os extratores ácidos e/ou salinos com o extrator água quente, indicando comportamento semelhante na determinação do B disponível.

Tabela 2 - Produção de matéria seca da parte aérea (MSPA) e teor foliar de B (Teor) na soja cultivada em casa de vegetação com solos de três localidades ${ }^{1}$.

\begin{tabular}{|c|c|c|c|c|c|c|}
\hline \multirow[t]{2}{*}{ Doses } & \multicolumn{2}{|c|}{ RQo-AG } & \multicolumn{2}{|c|}{ LVef-MS } & \multicolumn{2}{|c|}{ LVef-Lo } \\
\hline & MSPA & Teor & MSPA & Teor & MSPA & Teor \\
\hline $\mathrm{kg} \mathrm{ha}^{-1}$ & $\left(\right.$ g vaso $\left.^{-1}\right)$ & $\left(\mathrm{mg} \mathrm{kg}^{-1}\right)$ & $\left(\right.$ g vaso $\left.^{-1}\right)$ & $\left(\mathrm{mg} \mathrm{kg}^{-1}\right)$ & $\left(\right.$ g vaso $\left.^{-1}\right)$ & $\left(\mathrm{mg} \mathrm{kg}^{-1}\right)$ \\
\hline 0 & 3,69 & 59,22 & 1,83 & 45,22 & 2,15 & 30,32 \\
\hline 0,25 & 5,05 & 77,51 & 3,37 & 42,23 & 3,43 & 26,48 \\
\hline 0,5 & 9,49 & 76,51 & 3,50 & 58,80 & 4,26 & 26,10 \\
\hline 1,0 & 4,75 & 136,04 & 3,31 & 77,50 & 6,10 & 27,77 \\
\hline 2,0 & 2,03 & 215,45 & 2,39 & 114,78 & 7,85 & 42,13 \\
\hline 4,0 & 1,32 & 236,45 & 1,54 & 213,52 & 4,83 & 76,03 \\
\hline Teste F & $9,70 * *$ & $69,27 * *$ & $6,79 *$ & $1415,54 * *$ & $1,08^{\mathrm{NS}}$ & $48,57 * *$ \\
\hline
\end{tabular}

${ }^{1}$ Coeficientes seguidos de * e ** são significativos a 5\% e 1\% de probabilidade (teste F). ${ }^{\text {NS }}$ não significativo. LVef-Lo - LATOSSOLO VERMELHO Eutroférrico de Londrina (PR), LVef-MS - LATOSSOLO VERMELHO Eutroférrico de Mauá da Serra e RQo-AG NEOSSOLO QUARTZARÊNICO Órticos de Alto Garças (MT) (PR).

Tabela 3 - Coeficientes de correlação entre o B do solo extraído por água quente (B-AQ), Mehlich 1 (B-M1), KCl 1,0 mol $\mathrm{L}^{-1}(\mathrm{~B}-\mathrm{KCl}), \mathrm{Ca}\left(\mathrm{H}_{2} \mathrm{PO}_{4}\right)_{2} \cdot \mathrm{H}_{2} \mathrm{O} 0,1 \mathrm{~mol} \mathrm{~L}^{-1}\left(\mathrm{~B}-\mathrm{CaPO}_{4}\right), \mathrm{HCl} 0,05 \mathrm{~mol} \mathrm{~L}^{-1}(\mathrm{~B}-\mathrm{HCl})$ e Mehlich 3 (B-M3) com a produção de matéria seca, teor e conteúdo de $\mathrm{B}$, em soja cultivada em casa de vegetação com solos de três localidades ${ }^{1}$.

\begin{tabular}{|c|c|c|c|c|c|}
\hline \multirow[t]{2}{*}{ Extrator } & \multirow[t]{2}{*}{ Variável } & \multicolumn{4}{|c|}{ Localidade } \\
\hline & & RQo-AG & LVef-MS & LVef-Lo & Total \\
\hline \multirow[t]{3}{*}{ B-AQ } & Matéria seca $\left(\mathrm{g}\right.$ vaso $\left.^{-1}\right)$ & $0,76^{* *}(\mathrm{~L})$ & $0,76^{* *}(\mathrm{~L})$ & $0,14^{\mathrm{NS}}(\mathrm{Q})$ & $0,46^{\mathrm{NS}}(\mathrm{Q})$ \\
\hline & Teor $\left(\mathrm{mg} \mathrm{kg}^{-1}\right)$ & $0,86^{* *}(\mathrm{~L})$ & $0,96 * *(\mathrm{Q})$ & $0,58 *(\mathrm{Q})$ & $0,50 *(\mathrm{Q})$ \\
\hline & Conteúdo $\left(\mu \mathrm{g}_{\text {vaso }}{ }^{-1}\right)$ & $0,80 * *(\mathrm{Q})$ & $0,82 * *(\mathrm{~L})$ & $0,44^{\mathrm{NS}}(\mathrm{Q})$ & $0,11^{\mathrm{NS}}(\mathrm{Q})$ \\
\hline \multirow[t]{3}{*}{ B-M1 } & Matéria seca $\left(\right.$ g vaso $\left.^{-1}\right)$ & $-0,68 *(\operatorname{Exp})$ & $0,58 *(\mathrm{Q})$ & $0,33^{\mathrm{NS}}(\mathrm{L})$ & $0,60 *(\mathrm{~L})$ \\
\hline & Teor $\left(\mathrm{mg} \mathrm{kg}^{-1}\right)$ & $0,87 * *(\mathrm{~L})$ & $0,81 * *(\mathrm{~L})$ & $0,62 *(\mathrm{~L})$ & $0,51 *(\mathrm{Q})$ \\
\hline & Conteúdo ( $\mu \mathrm{g} / \mathrm{vaso})$ & $-0,07^{\mathrm{NS}}(\mathrm{L})$ & $0,58 *(\mathrm{Q})$ & $0,69 *(\mathrm{~L})$ & $0,11^{\mathrm{NS}}(\mathrm{L})$ \\
\hline \multirow[t]{3}{*}{$\mathrm{B}-\mathrm{KCl}$} & Matéria seca $\left(\mathrm{g} \mathrm{vaso}^{-1}\right)$ & $-0,65^{*}(\mathrm{~L})$ & $0,63^{*}(\mathrm{Q})$ & $0,54 *(\mathrm{Q})$ & $-0,46^{\mathrm{NS}}(\mathrm{L})$ \\
\hline & Teor $\left(\mathrm{mg} \mathrm{kg}^{-1}\right)$ & $0,92 * *(\mathrm{~L})$ & $0,96 * *(\mathrm{~L})$ & $0,83 * *(\mathrm{~L})$ & $0,86^{* *}(\mathrm{~L})$ \\
\hline & Conteúdo $\left(\mu \mathrm{g}_{\text {vaso }^{-1}}\right)$ & $0,48^{\mathrm{NS}}(\mathrm{Q})$ & $0,82 * *(\mathrm{Q})$ & $0,73 * *(\mathrm{~L})$ & $0,44^{\mathrm{NS}}(\mathrm{Q})$ \\
\hline
\end{tabular}

Continua... 
Tabela 3 - Continuação...

\begin{tabular}{cccccc}
\hline B-CaPO & Matéria seca $\left(\mathrm{g} \mathrm{vaso}{ }^{-1}\right)$ & $-0,65^{*}(\mathrm{Pot})$ & $0,61^{*}(\mathrm{Q})$ & $0,34^{\mathrm{NS}}(\mathrm{Q})$ & $-0,51^{*}(\mathrm{~L})$ \\
& Teor $\left(\mathrm{mg} \mathrm{kg}^{-1}\right)$ & $0,93^{* *}(\mathrm{Q})$ & $0,94^{* *}(\mathrm{~L})$ & $0,79 *(\mathrm{~L})$ & $0,63^{*}(\mathrm{~L})$ \\
& Conteúdo $\left(\mu \mathrm{g} \mathrm{vaso}^{-1}\right)$ & $0,21^{\mathrm{NS}}(\mathrm{Q})$ & $0,79^{* *}(\mathrm{Q})$ & $0,69^{*}(\mathrm{~L})$ & $0,03^{\mathrm{NS}}(\mathrm{L})$ \\
$\mathrm{B}-\mathrm{HCl}$ & Matéria seca $\left(\mathrm{g} \mathrm{vaso}^{-1}\right)$ & $-0,67^{*}(\mathrm{Pot})$ & $0,54^{*}(\mathrm{Q})$ & $0,32^{\mathrm{NS}}(\mathrm{Q})$ & $0,60^{*}(\mathrm{Exp})$ \\
& Teor $\left(\mathrm{mg} \mathrm{kg}^{-1}\right)$ & $0,93^{* *}(\mathrm{~L})$ & $0,84^{* *}(\mathrm{~L})$ & $0,83^{* *}(\mathrm{Q})$ & $0,85^{* *}(\mathrm{Q})$ \\
& Conteúdo $\left(\mu \mathrm{g} \mathrm{vaso}^{-1}\right)$ & $0,38^{\mathrm{NS}}(\mathrm{Q})$ & $0,60^{* *}(\mathrm{Q})$ & $0,72^{* *}(\mathrm{Q})$ & $0,42^{\mathrm{NS}}(\mathrm{Q})$ \\
$\mathrm{B}-\mathrm{M} 3$ & Matéria seca $\left(\mathrm{g} \mathrm{vaso}^{-1}\right)$ & $-0,79^{*}(\mathrm{Pot})$ & $0,62^{*}(\mathrm{Q})$ & $0,60^{*}(\mathrm{Q})$ & $-0,49^{\mathrm{NS}}(\mathrm{L})$ \\
& Teor $\left(\mathrm{mg} \mathrm{kg}^{-1}\right)$ & $0,93^{* *}(\mathrm{Q})$ & $0,95^{* *}(\mathrm{~L})$ & $0,82^{* *}(\mathrm{~L})$ & $0,82^{* *}(\mathrm{~L})$ \\
& Conteúdo $\left(\mu \mathrm{g} \mathrm{vaso}^{-1}\right)$ & $0,37^{\mathrm{NS}}(\mathrm{Q})$ & $0,69^{* *}(\mathrm{Q})$ & $0,73^{* *}(\mathrm{~L})$ & $0,53^{*}(\mathrm{~L})$ \\
\hline
\end{tabular}

${ }^{1}$ Coeficientes seguidos de * e ** são significativos a 5\% e $1 \%$ de probabilidade (Teste F) ${ }^{\mathrm{Ns}}$ não significativo; segundo os modelos linear (L), potência (Pot), exponencia] Ex) e quadrático (Q). AG - Alto Garças (MT), MS - Mauá da Serra (PR) e Lo - Londrina (PR).

Tabela 4 - Coeficientes de correlação linear entre os extratores água quente (B-AQ), Mehlich 1 (B-M1), $\mathrm{KCl} \mathrm{1,0} \mathrm{mol} \mathrm{L-1} \mathrm{(B-KCl),}$ $\mathrm{Ca}\left(\mathrm{H}_{2} \mathrm{PO}_{4}\right)_{2} \cdot \mathrm{H}_{2} \mathrm{O} 0,01 \mathrm{~mol} \mathrm{~L}^{-1}\left(\mathrm{~B}-\mathrm{Ca}^{\mathrm{D}} \mathrm{O}_{4}\right), \mathrm{HCl} 0,05 \mathrm{~mol} \mathrm{~L}^{-1}(\mathrm{~B}-\mathrm{HCl})$ e Mehlich $3(\mathrm{~B}-\mathrm{M} 3)^{1}$.

\begin{tabular}{cccccc}
\hline & $\mathrm{B}-\mathrm{AQ}$ & $\mathrm{B}-\mathrm{KCl}$ & $\mathrm{B}-$ Mehlich 3 & B-Mehlich 1 & $\mathrm{B}-\mathrm{CaPO}_{4}$ \\
\hline $\mathrm{B}-\mathrm{HCl}$ & $0,56^{*}$ & $0,83^{* *}$ & $0,63^{*}$ & $0,64^{*}$ & $0,64^{*}$ \\
$\mathrm{~B}-\mathrm{CaPO}_{4}$ & $0,80^{* *}$ & $0,82^{* *}$ & $0,34^{\mathrm{NS}}$ & $0,84^{* *}$ & \\
$\mathrm{~B}-$ Mehlich 1 & $0,78^{* *}$ & $0,60^{*}$ & $0,31^{\mathrm{NS}}$ & & \\
$\mathrm{B}-\mathrm{Mehlich} \mathrm{3}$ & $0,10^{\mathrm{NS}}$ & $0,62^{*}$ & & & \\
B-KCl & $0,64^{*}$ & & & & \\
\hline
\end{tabular}

* e ** são significativos a $5 \%$ e $1 \%$ de probabilidade (Teste F), ${ }^{\text {NS }}$ não significativo

\section{CONCLUSÕES}

Os extratores água quente e $\mathrm{KCl} 1,0 \mathrm{~mol} \mathrm{~L}^{-1}$ foram os mais eficientes em avaliar a disponibilidade de boro em Latossolo Vermelho Eutroférrico e Nesossolo Quartzarênico Órtico.

A aplicação de $0,5 \mathrm{~kg} \mathrm{ha}^{-1}$ de B no LVef de Mauá da Serra e RQo de Alto Garças proporcionam maiores produções de matéria seca, enquanto no LVef de Londrina foi com 2,0 $\mathrm{kg} \mathrm{ha}^{-1}$.

\section{REFERÊNCIAS BIBLIOGRÁFICAS}

ABREU, M.F.; ABREU, A.A.; ANDRADE, J.C. Determinação de boro em água quente, usando aquecimento com microonda. In: RAIJ, B. van; ANDRADE, J.C.; CANTARELLA, H.; QUAGGIO, J.A. (Eds.). Análise química para avaliação da fertilidade de solos tropicais. Campinas: Instituto Agronômico de Campinas, 2001. p.231-239.

ALVAREZ VENEGAS, V.H.; DIAS, L.E.; RIBEIRO JUNIOR, E.S.; SOZA, R.B.; FONSECA, C.A. Métodos de análises de enxofre em solos e plantas. Viçosa, MG: UFV, 2001. 131p.
BATAGLIA, O.C.; RAIJ, B. Eficiência de extratores na determinação de boro em solos. Revista Brasileira de Ciência do Solo, Campinas, v.14, p.25-31, 1990.

CASTRO, C.; OLIVEIRA, F.A. Nutrição e adubação do girassol. In: LEITE, R.M.V.B.C.; BRIGHENTI, A.M.; CASTRO, C. (Eds.). Girassol no Brasil. Londrina: CNPSO, 2005. p.317-374.

\section{EMPRESA BRASILEIRA DE PESQUISA} AGROPECUÁRIA. Manual de métodos de análise de solo. Rio de Janeiro: CNPS/EMBRAPA, 1997. 212p.

EVANS, C.E. Soil tests calibration. In: BROWN, J.R. (Ed.). Soil testing: sampling, correlation, calibration and interpretation. Madison: Soil Science Society of America, 1987. p.23-29.

FEHR, W.A.; CAVINESS, C.E. Stages of soybean development. Ames: Iowa State University, 1977. 11p. (Iowa Agriculture Experimental Station Bulletin, 80). 
GOLDBERG, S. Reactions of boron with soils. In: DELL, B.; BROW, P.H.; BELL, R.W. (Eds.). Boron in soil and plants: reviews. Dordrecht: Kluwer Academic, 1997. p.35-48.

GUPTA, U. A simplified method for determining hot water soluble in Podzol soils. Soil Science, Baltimore, v.103, p.424-429, 1967.

MALAVOLTA, E. Elementos de nutrição mineral de plantas. São Paulo: Ceres, 1980. 251p.

MALAVOLTA, E.; VITTI, G.C.; OLIVEIRA, S.A. Avaliação do estado nutricional das plantas: princípios e aplicações. Piracicaba: Associação Brasileira para Pesquisa da Potassa e do Fosfato, 1997. 319p.

MEHLICH, A. Mehlich 3 soil test extractant; a modification of Mehlich 2 extractant. Communications in Soil Science and Plant Analysis, New York, v.15, p.1409-1416, 1984.

MEHLICH, A. New extractant for soil test evaluation of phosphorus, potassium, magnesium, calcium, sodium, manganese and zinc. Communications in Soil Science and Plant Analysis, New York, v.9, p.477-492, 1978.
MOREIRA, A.; CASTRO, C. Extratores ácidos e sais na determinação da disponibilidade de boro no solo. Manaus: Embrapa Amazônia Ocidental, 2006. 5p. (Comunicado Técnico, 36).

PIMENTEL GOMES, F.; GARCIA, C.H. Estatística aplicada a experimentos agronômicos e florestais. Piracicaba: FEALQ, 2002. 309p.

PONNAMPERUMA, F.N.; CAYTON, M.T.; LANTIN, R.S. Dilute hydrochloric acid as an extractant for available zinc, cooper and boron in rice soils. Plant and Soil, The Hague, v.61, p.297-310, 1981.

SILVA, F.R.; FERREYRA, F.F. Avaliação de extratores de boro em solos do Estado do Ceará. Revista Brasileira de Ciência do Solo, Campinas, v.22, p.471-478, 1998.

\section{TECNOLOGIAS DE PRODUÇÃO DE SOJA. Região} central do Brasil 2007. Londrina: Embrapa Soja; Embrapa Cerrados; Embrapa Agropecuária Oeste, 2006. 225p.

YAMADA, T. Boro: será que estamos aplicando a dose suficiente para o adequado desenvolvimento das plantas? Informações Agronômicas, Piracicaba, n.90, p.1-5, 2000. 